\title{
Non-Operative Treatment in Children and Adolescents with Atlantoaxial Rotatory Subluxation
}

\author{
Mert Çiftdemir, Cem Çopuroğlu, Mert Özcan, Ayşe Ovul Ulusam, Erol Yalnız \\ Department of Orthopaedics and Traumatology, Faculty of Medicine, Trakya University, Edirne, Turkey
}

\begin{abstract}
Objective: Atlantoaxial rotatory subluxation (AARS) is an uncommon lesion seen mainly in children and adolescents. A retrospective analysis of 12 patients with AARS treated non-operatively is presented in this study.

Material and Methods: Twelve patients with AARS who were treated non-operatively were evaluated retrospectively in terms of recent trauma and respiratory tract infection history, accompanying injuries, radiological findings, duration of symptoms, amount and duration of traction and clinical results of the treatment.

Results: Traumatic AARS was present in 8 patients. No evidence of trauma was found in 4 patients. Type I subluxations in 10 and type II subluxations in 2 patients were found according to the Fielding and Hawkins classification. All patients were treated using bed-side mentooccipital tractions. The mean duration of bed-side mentooccipital traction was 3,75 days and the mean amount of load was $1.8 \mathrm{~kg}$. All patients were kept in Philadelphia collars for additional 3 weeks after the clinical recovery. No limitations and pain in head movements were present in any patient at the sixth month follow-up examinations.
\end{abstract}

Conclusion: Paediatric patients with neck pain and torticollis should be investigated concerning trauma and recent respiratory tract infection history in order to avoid any delay in diagnosis of a possible AARS.

Key Words: Atlanto-axial joint, torticollis, neck pain

\section{Introduction}

Atlantoaxial rotatory subluxation (AARS) is an incomplete dislocation of the inferior atlantal and superior axial articular facets. It is generally seen in the paediatric patient population following upper respiratory tract infections, tonsillectomies, head trauma and induction for general anaesthesia (1).

Patients with AARS usually present with painful torticollis and limited neck motion several days after an upper respiratory tract infection or a head trauma. Early diagnosis plays an important role in the management of AARS. As mainly seen in the paediatric population, parents of the patients often ignore this painful condition assuming that it is a crick. Increasing pain and marked deformity leads the parents of the patient to refer to a physician after a period of delay.

Conservative treatment is successful among the patients with early diagnosis. Delayed diagnosis is often seen even in the patients with acute head trauma. Persistent torticollis, neurological compromise and poor results may be seen in the cases with a delayed diagnosis. In this study we aimed to emphasize the importance of early diagnosis for AARS in patients with or without evidence of trauma. In order to highlight the key points concerning early diagnosis and conservative treatment, a retrospective analysis of 12 patients with
AARS treated non-operatively between 2000 and 2010 are presented in this study.

\section{Material and Methods}

Four female and 8 male patients with a mean age of 11.5 (4-17) with AARS, treated non-operatively between 2000 and 2010, were evaluated in this retrospective study. All 12 patients presented with acute neck pain and torticollis at admittance. There was no evidence of trauma in 4 patients. Three of these patients had a history of antibiotic use due to upper respiratory tract infections within the last 3 weeks before the symptoms. Injury mechanisms of the 8 patients with traumatic AARS were analysed. The injury mechanisms were motor vehicle collisions in 2 patients, blows to the head in 3 patients, sports injuries in 2 patients and a suicide attempt by hanging in one patient. Fracture of the femoral shaft in one patient, a clavicle fracture in one patient, and tibia fracture in one patient were found as additional injuries in 3 patients. AARS were diagnosed in all patients with open mouth atlantoaxial view radiographs and axial CT scans. Loss of symmetry between lateral masses of the atlas and odontoid process in open mouth atlantoaxial view radiographs (Figure 1) and axial CT scans was the radiological inclusion criteria in our study. 
Atlantoaxial rotatory subluxations in all patients were evaluated according to the Fielding and Hawkins classification (2).

All patients were hospitalized and custom-made bedside mentooccipital head-halter tractions were applied (Figure 2). Patients' age and weights were used to determine the weights used for mentooccipital traction. Weights of $1 \mathrm{~kg}$ were used for traction in the patients younger than 10 years of age and weighing less than $25 \mathrm{~kg}$, while weights of $2 \mathrm{~kg}$ were used in the patients older than 10 years of age and weighing more than $25 \mathrm{~kg}$. There was an exception for one adolescent patient who had non-traumatic AARS. The patient was admitted to hospital after 3 weeks of neck pain. Mentooccipital headhalter traction was applied with $4 \mathrm{~kg}$ for this particular patient. Neurological conditions with neck pain and range of motion were examined and recorded twice daily for all patients. Mentooccipital tractions were terminated and Philadelphia hard collars were applied in the patients whose symptoms had re-

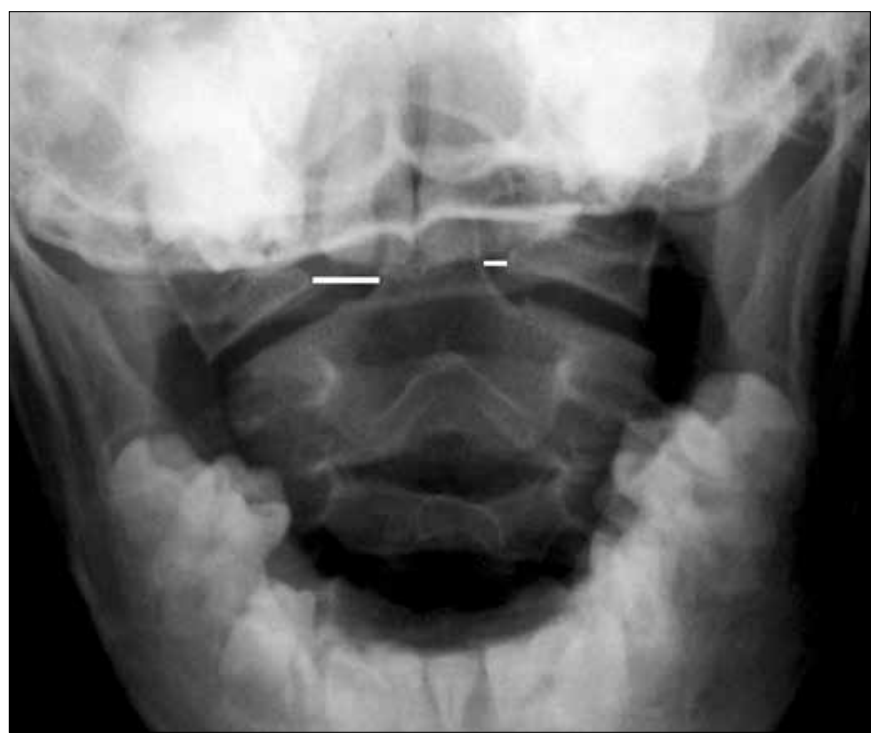

Figure 1. Loss of symmetry between lateral masses of the atlas and odontoid process in open mouth atlantoaxial view radiograph

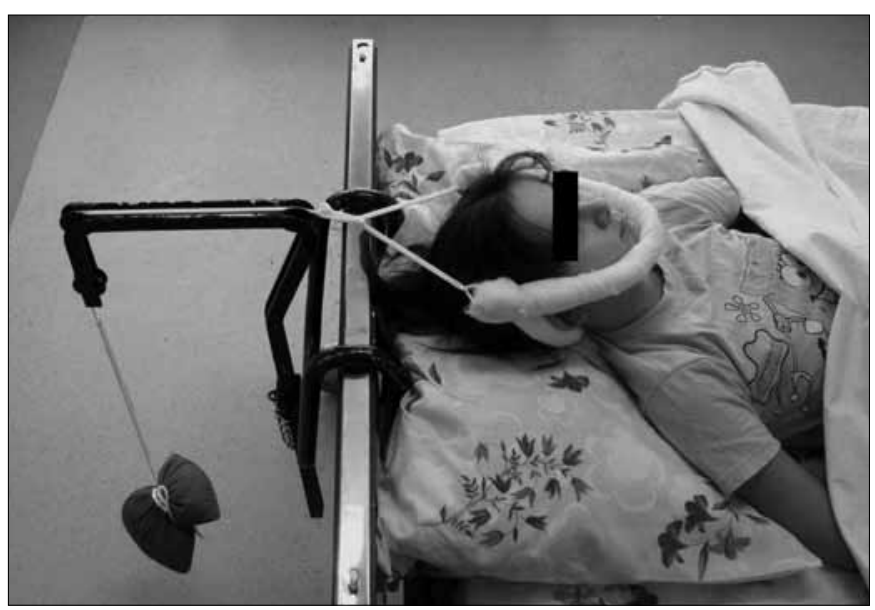

Figure 2. Custom made bedside mentooccipital head-halter traction gressed and pain-free neck range motions were obtained. The patients were then discharged from hospital with Philadelphia hard collars and recalled for periodic controls.

\section{Results}

The reasons for admittance were pain and loss of neck mobility in all patients. Eight of the 12 patients in our study were admitted to our emergency unit after acute traumatic events. The mean duration of symptoms was 4.8 (1-20) days in our study group. Neck pain, sternocleidomastoideus and paravertebral muscular spasm, torticollis and limited head movements were determined at the initial physical examination in all patients. No neurological deficits were found in any patients at admittance except for the patient with the suicide attempt. The patient was unconscious due to hypoxia. It was found that the patient was neurologically intact at the definitive examination on the next day in the intensive care unit. Type I subluxations were found in 10 and type II subluxations were found in 2 patients according to the Fielding and Hawkins classification (2).

Custom-made bedside mentooccipital head-halter tractions were applied to all patients. The neurological status, pain and head movements in all patients were examined twice daily. Tractions were terminated and Philadelphia hard collars were applied to the patients who regained pain-free head rotations, whose muscle spasms were resolved and normal tonus of the bilateral sternocleidomastoideus muscles were achieved. The mean duration of bedside mentooccipital traction in all patients was $3.75(2-5)$ days and the mean weight used for traction was 1.8 (1-4) kg. Pain-free head rotations and normal range of motion at the cervical vertebrae were noted and collars were removed after 3 weeks follow-up in all patients. The patients were followed up for 6 months. No limitation in head rotations, no pain and no neurological abnormality in any patient were observed at the end of the follow-up period.

\section{Discussion}

The atlantoaxial joint is a unique articulation, which is the cornerstone of the upper cervical spine and allows a large degree of the head rotation. The superior and inferior articular facets of the atlas differ from each other in terms of their geometrical designs. The occipital condyles nestle in the concavely designed superior articular facets of the atlas. Inferior articular facets of the atlas and superior articular facets of the axis are both convex in design. In opposition to the atlantoooccipital articulation, which allows weight bearing and some flexion-extension, atlantoaxial articulation allows nearly half of the axial rotation of the entire cervical spine (3).

Stability of the atlantoaxial joint, which has such a wide range of motion, is provided by both soft tissues and bony structures. Alar ligaments, apical ligament and the transverse ligament are the soft tissue components of the atlantoaxial stability. The facet joints and the odontoid process are the bony structures supporting the atlantoaxial joint. Dvorak has shown that the transverse ligament, which is twice as strong as the alar ligaments, is the main stabilizer of this joint (4). 
Fielding and Hawkins classified the rotatory subluxations of the atlantoaxial joint and described 4 lesion types (2). This classification is based on the centre of rotation and the anterior displacement of the atlas on axis at the sagittal plane (Table 1). Harty et al. have stated that type I and type II lesions are predominantly seen in children and are often associated with delayed diagnosis (5). The transverse ligament, which is the main stabilizer of the atlantoaxial joint, is intact in type I lesions. The transverse ligament is thought to be injured in type II, III and IV lesions according to the Fielding and Hawkins classification.

A flexion-extension type injury with associated rotational force vectors may cause AARS (3). AARS is not common in the adult population and usually accompanies lateral mass or facet joint fractures of $\mathrm{C} 1$ and C2. AARS in childhood and adult ages differ from each other in terms of pathophysiology and biomechanics. The children are more prone to AARS because of a relatively larger head diameter, underdeveloped neck muscles, and more horizontally oriented and convex designed facet joints with more elastic facet joint capsules (6).

Non-traumatic atlantoaxial subluxation following nasopharyngeal inflammatory conditions in children is called Griesel's syndrome. It is predominantly seen in children between 3 and 12 years of age. The cause of Grisel's syndrome is thought to be a distension of the facet joint capsules and stretching of the transverse ligament because of the inflammatory process affecting the synovium, the lymphatic vessels and the periodontoideal venous plexus (7). Griesel hypothesized that sustained muscle spasms of the paravertebral and cervical musculature lead to torticollis in an attempt to decompress the inflammated tissue (7).

Usually, diagnosis is not easy with plain radiographs in AARS. While open mouth atlantoaxial view radiographs and dynamic plain radiographs are sometimes helpful, the most beneficial method is the computerized tomography (CT) examination. The disturbance of the symmetry between the lateral masses of the atlas and the odontoid process in the open mouth atlantoaxial view is thought to be a rotatory subluxation. Rotation of C1 vertebra and the relation between the anterior arcus of the atlas and the odontoid process is best evaluated in CT scans (Figure 3). The anterior displacement of the atlas more than 3 $\mathrm{mm}$ in children and more than $5 \mathrm{~mm}$ in adults is considered as evidence of a transverse ligament injury.
Stability of the atlantoaxial complex and difficulties in diagnosis are the main determinants for the type of the treatment in AARS. Open reduction is often indicated for the cases with delayed diagnosis because of the irreducible subluxations. The mean duration between the symptoms and the diagnosis was 11.6 months among the 17 patients reported in the original article of Fielding et al. Thirteen of the 17 patients were treated with atlantoaxial fusion in this series (2). Crossman et al. have treated 13 paediatric cases with irreducible atlantoaxial rotatory fixations with open reduction and halo immobilization (1). The authors have observed an improvement in the head positions, sagittal range of motions and facial asymmetries of the patients who underwent open reduction and halo immobilization. In this study, the authors have also observed reduction and asymmetry in the axial rotation of the surgically treated patients compared with that in control individuals. The patients treated with open reduction and halo immobilization had a delayed diagnosis ranging from 3 to 36 months in this study.

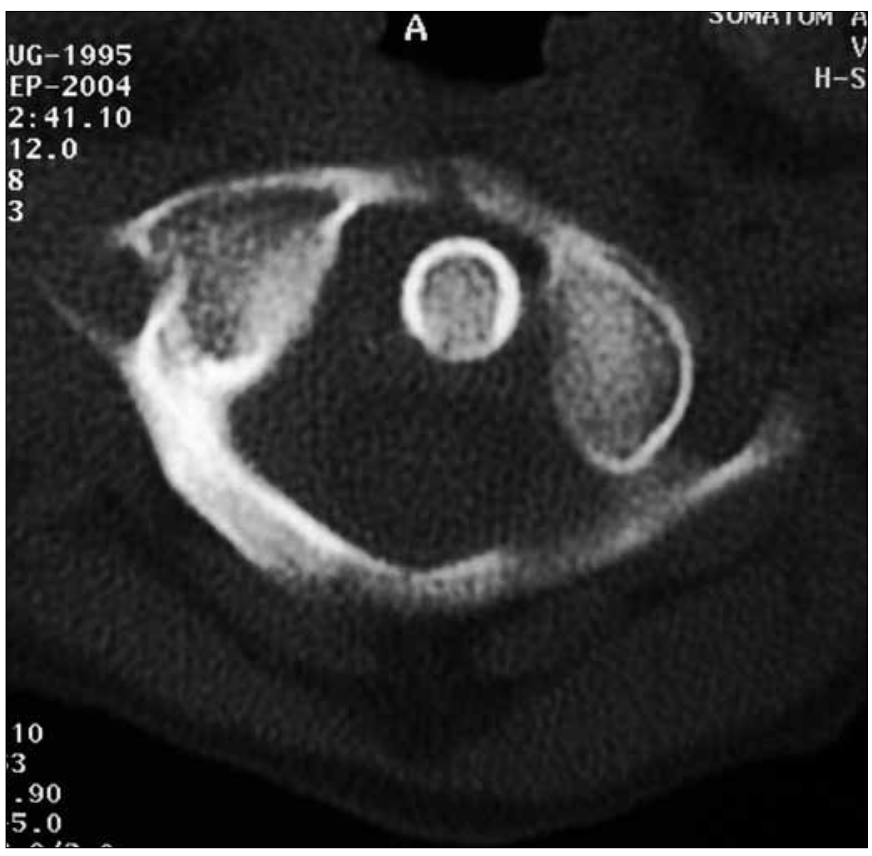

Figure 3. Rotation of C1 vertebra and the relation between the anterior arcus of the atlas and the odontoid process in axial CT scan

Table 1. Atlantoaxial rotatory fixation classification according to Fielding and Hawkins (2)

\section{Type I}

Rotatory fixation without anterior displacement of the Atlas

Type II

Rotatory fixation with anterior displacement

Type III

Rotatory fixation with anterior displacement more than $5 \mathrm{~mm}$

Type IV

Rotatory fixation with posterior displacement
Odontoid acting as the pivot, no anterior displacement

One lateral articular process acting as the pivot, anterior displacement 3 to $5 \mathrm{~mm}$

Rotatory fixation with anterior displacement more than $5 \mathrm{~mm}$, displacement of both atlantal lateral masses

Rotatory fixation with posterior displacement 
Successful results were achieved with non-operative treatment in the patients with intact transverse ligament and preserved joint stability. Non-operative treatment consisted of anti-inflammatory drugs and head traction to eliminate muscle spasm. Elimination of the muscle spasm leads to reduction of the rotatory subluxation. Phillips et al. (8) treated 23 paediatric patients with AARS and reported that success of closed reduction is correlated with the duration of torticollis. Three of the 7 patients, who had torticollis for more than 1 month in the series, were treated with atlantoaxial arthrodesis. Successful results were reported by Subach et al. in the non-operative treatment of AARS in 20 paediatric patients with a mean period of 11.2 days before the diagnosis (9).

\section{Conclusion}

AARS are mainly seen in the paediatric population after minor head trauma or following a respiratory tract infection. Torticollis and decreased cervical range of motion are the major clinical findings. These clinical findings may be indistinct at the early period and this leads to diagnostic delay. These subluxations often reduce spontaneously in a few days, but sometimes appear in a spectrum ranging from slight restriction in cervical range of motion to rigid and permanent torticollis, facial asymmetry and even sudden death.

Non-operative treatment is successful in the patients with an early diagnosis. It is known that unsuccessful results are correlated with the duration of symptoms. Surgical treatment options are open reduction with temporary fixation and instrumented or non-instrumented atlantoaxial fusion. Atlantoaxial fusion results in a permanent loss of nearly $50 \%$ of the axial head rotation. Open reduction with temporary fixation does not yield better results than the non-operative treatment.

Early elimination of AARS in acute and subacute neck pain and torticollis in paediatric patients has a significant impor- tance. Paediatric patients with neck pain and torticollis should be investigated concerning trauma and recent respiratory tract infection history in order to avoid any delay in diagnosis of a possible AARS.

\section{Conflict of Interest}

No conflict of interest was declared by the authors.

\section{References}

1. Crossman JE, David K, Hayward R, Crockard HA. Open reduction of pediatric atlantoaxial rotatory fixation: Long term outcome study with functional measurements. J Neurosurg (Spine 3) 2003;100:235-40.

2. Fielding JW, Hawkins RJ. Atlanto-axial rotatory fixation. (Fixed rotatory subluxation of the atlanto-axial joint). J Bone Joint Surg Am 1977;59:37-44.

3. Vaccaro AR, Milam VI RA, Bassewitz HL, Herkowitz HN, Kubeck JP. Atlantoaxial Rotatory Instability. In: Vaccaro AR, ed. Fractures of the Cervical, Thoracic and Lumbar Spine. New York-Basel, etc: Marcel Dekker Inc., 2003: 127-37.

4. Dvorak J, Schneider E, Saldinger P, Rahn B. Biomechanics of the craniocervical region: the alar and transverse ligaments. J Orthop Res 1988;6:452-61. [CrossRef]

5. Harty JA, Lenehan B, O'Rourke SK. Odontoid lateral mass asymmetry: do we over-investigate? Emerg Med J 2005;22:625-7. [CrossRef]

6. Missori P, Miscusi M, Paolini S, DiBiasi C, Finocchi V, Peschillo S, et al. A C1-2 locked facet in a child with atlantoaxial rotatory fixation. Case report. J Neurosurg 2005;103(6 Suppl):563-6.

7. Wetzel FT, La Rocca H. Grisel's Syndrome. Clin Orthop Relat Res 1989;240:141-51.

8. Philips WA, Hensinger RN. The management of rotatory atlantoaxial subluxation in children. J Bone Joint Surg Am 1989;71:664-8.

9. Subach BR, McLaughlin MR, Albright AL, Pollack IF. Current management of pediatric atlantoaxial rotatory subluxation. Spine 1988;23:2174-9. [CrossRef] 